\title{
Salud bucal y funcionamiento familiar en personas del municipio Plaza de la Revolución de La Habana, Cuba
}

\author{
Oral Health and Family Functioning among Individuals from the Plaza de la Revolución \\ Municipality of Havana, Cuba
}

Fecha de recepción: 05-03-2018 | Fecha de aceptación: 08-11-2018

\author{
ISIS BÁRBARA HERRERA LóPEZ ${ }^{a}$ \\ Facultad de Estomatología de La Habana "Raúl González Sánchez", La Habana, Cuba. \\ isisherrera@infomed.sld.cu. https://orcid.org/0000-0002-0364-750X \\ ${ }^{a}$ Correspondencia: $\underline{\text { isisherrera@infomed.sld.cu }}$ \\ doi: https://doi.org/10.11144/Javeriana.uo37-79.sbff
}

Cómo citar: Herrera López IB. Salud bucal y funcionamiento familiar en personas del municipio Plaza de la Revolución de La Habana, Cuba. Univ Odontol. 2018 jul-dic; 38(79). https://doi.org/10.11144/Javeriana.uo37-79.sbff

\section{RESUMEN}


Antecedentes: La familia constituye el espacio por excelencia para el desarrollo de la identidad humana y es el primer grupo de socialización del individuo. En ella la persona adquiere sus primeras experiencias, hábitos, valores y concepciones del mundo, incluyendo la salud. Objetivo: Analizar el estado de salud bucal de las personas en relación con el funcionamiento familiar. Métodos: Se realizó un estudio descriptivo con 84 sujetos de 20 familias pertenecientes a un consultorio odontológico del municipio Plaza de la Revolución de la ciudad de La Habana, Cuba. Los pacientes contestaron encuestas de funcionamiento familiar y hábitos bucales y recibieron evaluación clínica domiciliaria $\left(\mathrm{p}=0,05, \mathrm{Chi}^{2}\right)$. Resultados: Predominó el edentulismo (53,57 \%) que fue más común en personas de familias disfuncionales. El 70,24 \% tenía mala higiene bucal. La mayoría de quienes tenían buena higiene bucal (88 \%) pertenecían a familias funcionales. El $51,19 \%$ se cepillaba los dientes solo dos veces al día, principalmente en aquellos de familias disfuncionales. 22,62 \% de las personas informó no consumir alimentos azucarados, la mayoría de ellos de familias funcionales. El 82,05 \% acudía al estomatólogo solamente cuando tenía una urgencia y eran todos de familias disfuncionales. Conclusión: El funcionamiento familiar se asoció a la salud bucal de las personas estudiadas. Se recomienda crear programas educativos de salud bucal con un enfoque familiar.

\section{Palabras clave}

caries dental; epidemiología; enfermedad periodontal; familia; funcionamiento familiar; hábitos bucales; maloclusiones

\section{Áreas temáticas}

atención primaria; epidemiología oral; odontología 


\section{ABSTRACT}

Background: Family is the space par excellence for the development of human identity and is a person's first socialization group. People have their first experiences, habits, values, and world perceptions, including health, within the family core. Purpose: To analyze oral health status of individuals regarding family functioning. Methods: A descriptive study with 84 persons of 20 families who belonged to the dental office of the Plaza de la Revolution municipality in Havana Cuba was carried out. Patients responded family functioning and oral habits surveys and underwent at-home clinical evaluations $\left(\mathrm{p}=0.05, \mathrm{Chi}^{2}\right)$. Results: Edentulism was predominant (53.57 \%) mainly among individuals from dysfunctional families. $70.24 \%$ had poor oral hygiene; however, most of those with good oral hygiene $(88.00 \%)$ belonged to functional families. 51.19 $\%$ only brushed their teeth twice a day, mainly from dysfunctional families. $22.62 \%$ of patients reported not consuming sugary foods, most of whom belonged to functional families. $82.05 \%$ informed seeing the dentist only when they had an dental emergency, all of them from dysfunctional families. Conclusion: Family functioning showed association with oral health status among the individuals studied. It is advisable to create family-focused preventive oral health programs.

\section{Keywords}

dental caries; epidemiology; family; family functioning; malocclusions; oral habits; periodontal diseases

\section{Thematic fields}


dentistry; oral epidemiology; primary health care

\section{INTRODUCCIÓN}

La salud, como categoría individual y social, puede abordarse con diversas estrategias de intervención, encaminadas al logro de acciones de prevención en los diferentes grupos sociales de la comunidad, con el fin de fomentar modos y estilos de vida saludables para sus miembros, donde la familia juega un papel importantísimo. Incluso se ve a la familia como uno de los grupos sociales de vital importancia en el terreno de la salud (1-4).

La familia es la instancia de intermediación entre el individuo y la sociedad. Constituye el espacio por excelencia para el desarrollo de la identidad, y es el primer grupo de socialización del individuo. Es en la familia donde la persona adquiere sus primeras experiencias, valores y concepciones del mundo incluyendo la salud. La familia aporta al individuo las condiciones para un desarrollo sano de la personalidad o, en su defecto, es la principal fuente de trastornos emocionales (1-4). La psicóloga Patricia Arés Muzio (4) define a "la familia como la unión de personas que comparten un proyecto vital de existencia común, que se quiere duradero, en el que se generan fuertes sentimientos de pertenencia a dicho grupo, existe un compromiso personal entre sus miembros y se establecen intensas relaciones de intimidad, reciprocidad y dependencia" (5-8). El ambiente familiar es de importancia crítica, por que en él se sitúan muchos episodios de interacción, de educación interactiva, a través de los cuales, la familia va proporcionando andamiajes al desarrollo infantil y dando contenido a su evolución (1-9). 
La familia constituye la unidad de reproducción y mantenimiento de la especie humana y en ese sentido es el elemento que sintetiza la producción de la salud a escala microsocial. En ella se satisfacen las necesidades que están en la base de la conservación, fomento y recuperación de la salud. Cumple funciones importantes en el desarrollo biológico, psicológico y social del individuo, encargada de la formación y desarrollo de la personalidad y asegura, junto a otros grupos sociales, la socialización y educación de este para su inserción en la vida social y la transmisión generacional de valores culturales, ético - morales y espirituales (2, 4, 6, 8, 10-12). En su seno se forman los motivos y hábitos relacionados con el comportamiento implicado en la salud, se gestan procesos que actúan como protectores de salud o desencadenantes de la enfermedad, y se desarrollan recursos de apoyo altamente significativo y efectivo $(1,2,4,5,10,12)$.

El funcionamiento familiar resulta un factor predisponente o coadyuvante en la producción y curso de diversas enfermedades y problemas de salud en sus integrantes $(2,4,5,10,12)$. Una familia con un funcionamiento adecuado, o familia funcional, puede promover el desarrollo integral de sus miembros y lograr el mantenimiento de estados de salud favorables en éstas. Así como también una familia disfuncional o con un funcionamiento inadecuado, debe ser considerada como factor de riesgo, al propiciar la aparición de síntomas y enfermedades en sus miembros (4-7). A la hora de hablar de funcionamiento familiar encontramos que no existe un criterio único de los indicadores que lo miden. Algunos autores plantean que la familia se hace disfuncional, cuando no se tiene la capacidad de asumir cambios, es decir, cuando la rigidez de sus reglas le impide ajustarse a su propio ciclo y al desarrollo de sus miembros. Otros autores señalan como características disfuncionales la incompetencia intrafamiliar y el incumplimiento de sus funciones básicas $(2,4,5,10,12)$. 
Si la familia establece estrategias disfuncionales ante las situaciones de cambio, como la rigidez y la resistencia, esto provoca una enquistación de los conflictos y por tanto, comienzan a aparecer síntomas que atentan contra la salud y el desarrollo armónico de sus miembros. Por lo tanto, la principal característica que debe tener una familia funcional es que promueva un desarrollo favorable a la salud para todos sus miembros, para lo cual es imprescindible que tenga: jerarquías claras, límites claros, roles claros y definidos, comunicación abierta y explícita, y capacidad de adaptación al cambio (10, 12-14).

Cada día existen más profesionales de la salud que consideran que los problemas de salud de un individuo no son solo un problema individual, sino también un problema familiar y su solución no solo involucra al enfermo sino a su familia. Dentro de la tríada ecológica, la familia constituye parte esencial del medio ambiente, donde el agente encuentra condiciones favorables o desfavorables a su acción, ante lo cual el huésped se halla protegido o desprotegido según el apoyo familiar. Podemos decir que la familia es en sí misma una tríada ecológica, aún hoy en parte desconocida, y por lo tanto con problemas de manejo por parte del estomatólogo $(13,14)$.

Se podría mencionar múltiples roles de la familia en el proceso salud-enfermedad, como por ejemplo que actúa indirectamente en la relación estomatólogo-niño, pues ofrece experiencias fundamentales, entre las cuales destacamos las aptitudes para con él (miedo subjetivo al tratamiento estomatológico), la profilaxis y el tratamiento bucal. Las actitudes de cada uno de los padres en cuanto a la higiene bucal y el comportamiento ante las acciones estomatológicas es 
decisivo sobre todo en edades tempranas, donde el niño imita todas las acciones de ellos, lo toman como patrón a seguir (10-15).

En el caso de la caries dental que es una enfermedad de alta prevalencia a nivel mundial, con más del $90 \%$, dentro de su multicausalidad se destacan aspectos del estilo de vida familiar, tales como: ingestión elevada de alimentos azucarados en la dieta diaria desde edades tempranas; pobre consumo de frutas y vegetales que aportan vitaminas y minerales tanto en las embarazadas como en los niños pequeños debido a una falta de cultura alimenticia; deficiente higiene bucal relacionada con un incorrecto cepillado dental (frecuencia y forma), por la falta de orientación de fisioterapia bucal desde el embarazo, etc. (15-22).

Con respecto a la enfermedad periodontal, los factores asociados incluyen deficiente higiene bucal, condiciones socioeconómicas desfavorables y factores sociales como el medio familiar (15-22). La enfermedad periodontal y la caries dental se encuentran en estrecha relación con la dieta ya que existen elementos dietéticos que promueven o disminuyen el desarrollo de estas, por ejemplo el carácter físico de los alimentos es un elemento de alto capaz de estimular los tejidos periodontales, barrer los restos adosados en los dientes, bacterias y células epiteliales descamadas. Una dieta consistente, capaz de estimular la encía durante la elaboración del bolo alimenticio, ayuda a aumentar la queratinización de esta, se hace más resistente a los embates del medio, y además estimula la circulación sanguínea de la encía y evita el éxtasis venoso (20,23-25).

En el caso de las maloclusiones o desarmonías oclusales, son catalogadas como un problema de salud ya que afecta entre el $54 \%$ al 78,8 \% de la población, en muchas ocasiones pueden ser 
prevenidas, si en la familia se ponen en práctica las actividades de promoción y prevención de salud desde que la mujer está embarazada, evitando que se instauren hábitos deformantes en el recién nacido como el biberón, el chupete y la succión digital; aspectos de la dieta como consistencia, composición de la misma e incentivando otros aspectos positivos para la salud como la lactancia materna $(26,27-31)$.

La succión digital, queilofagia, onicofagia y el bruxismo tienen dentro de sus causas un componente psíquico muy importante, pues por lo general se encuentran asociados a tensión, temor, inseguridad, conflictos emocionales insuperables dentro y fuera del núcleo familiar. Otros hábitos como el empuje lingual y la respiración bucal quedan como consecuencia de enfermedades respiratorias altas, o como secuela de otro hábito perjudicial (la succión digital puede dejar el hábito de empuje lingual). Sin embargo, a veces, en el núcleo familiar hay algún miembro que presenta algunos de estos y no le dan importancia alguna, ni tan siquiera acuden al médico para saber la causa que lo provoca (31- 35). Todos ellos de una forma u otra pueden producir alteraciones en el crecimiento y desarrollo fisiológico de las estructuras del aparato estomatognático, causando diferentes tipos de maloclusiones (31).

Cuando se produce el desdentamiento se traduce como el fracaso o poca importancia por parte de la familia y sus integrantes en implementar las acciones de promoción, prevención y de educación para la salud, ya que es el resultado de la acción de los diversos problemas bucales mencionados anteriormente. A esto se le suman las concepciones erróneas acerca de la relación directa e inevitable entre la pérdida dentaria y el avance de la edad, así como la presencia de hábitos incorrectos heredados en la familia como la mala higiene bucal, consumo excesivo de azúcares y 
el temor o miedo al tratamiento estomatológico que le impide acudir con regularidad para una revisión periódica $(15,32,33)$.

Existen múltiples estudios sobre familia y funcionamiento familiar, pero abordados desde otras aristas: jurídico, económico, psicológico, genético, etc.; sin embargo no ha sido enfocada como factor de riesgo sobre la salud bucal de cada uno de sus integrantes. Por lo tanto con esta investigación se pretende cambiar la forma de enfocar el trabajo comunitario del Estomatólogo General Integral hacia las familias según su funcionamiento familiar, planteándose la siguiente interrogante: ¿Existirá relación entre el funcionamiento familiar y la salud bucal de sus integrantes? Para dar respuesta a la misma se planteó como objetivo general analizar el estado de salud bucal de las personas en relación con el funcionamiento familiar. Los objetivos específicos fueron analizar la relación entre el funcionamiento familiar y la presencia o ausencia de enfermedades bucales, hábitos bucales dañinos, la higiene bucal, la ingestión de alimentos azucarados y la frecuencia y motivos de las visitas a la consulta estomatológica.

\section{MATERIALES Y MÉTODOS}

Se realizó un estudio descriptivo con 84 personas de 20 familias pertenecientes al consultorio 45 de la Policlínica 19 de Abril, municipio Plaza de la Revolución, de la Habana Cuba. El universo estuvo constituido por 724 individuos distribuidos en las 147 familias del consultorio mencionado, la muestra fueron los 84 miembros distribuidos en 20 familias, dicha muestra no probabilística se obtuvo por criterio de expertos. A cada uno de los integrantes se le aplicó un test de funcionamiento familiar (anexo 1), se le realizó un examen intrabucal en sus hogares y para clasificar la modalidad 
de la higiene bucal se aplicó el Índice de Love (anexo 2). La información obtenida fue recogida en una planilla confeccionada al efecto por el investigador (anexo 3). Todos los datos obtenidos fueron procesados digitalmente a través de programas estadísticos, en caso de relacionar dos ó más variables cualitativas se utilizó el estadígrafo Chi cuadrado. Para hacer más comprensibles los datos se confeccionaron gráficos.

\section{RESULTADOS}

De las personas incluidas en el estudio, 33 (39.29\%) pertenecían a familias funcionales, y 51 $(60.71 \%)$ a familias disfuncionales. Esta clasificación se basó en el test de funcionamiento familiar.

De las 84 personas distribuidas en las familias estudiadas (20 familias) se observó que el desdentamiento fue el problema de salud más frecuente, con 45 individuos para un $53.57 \%$, muy seguido de la caries dental, representada por 35 personas, para un $41.67 \%$. Hubo más afectados en las familias disfuncionales, observándose los porcientos más elevados. Solo el $19.05 \%$ resultaron sanos y todos pertenecían a familias funcionales. 
FIGURA 1

DISTRIBUCIÓN DE INDIVIDUOS, SEGÚN FUNCIONAMIENTO FAMILIAR Y LA PRESENCIA O AUSENCIA DE PROBLEMAS Y/O ENFERMEDADES BUCALES.

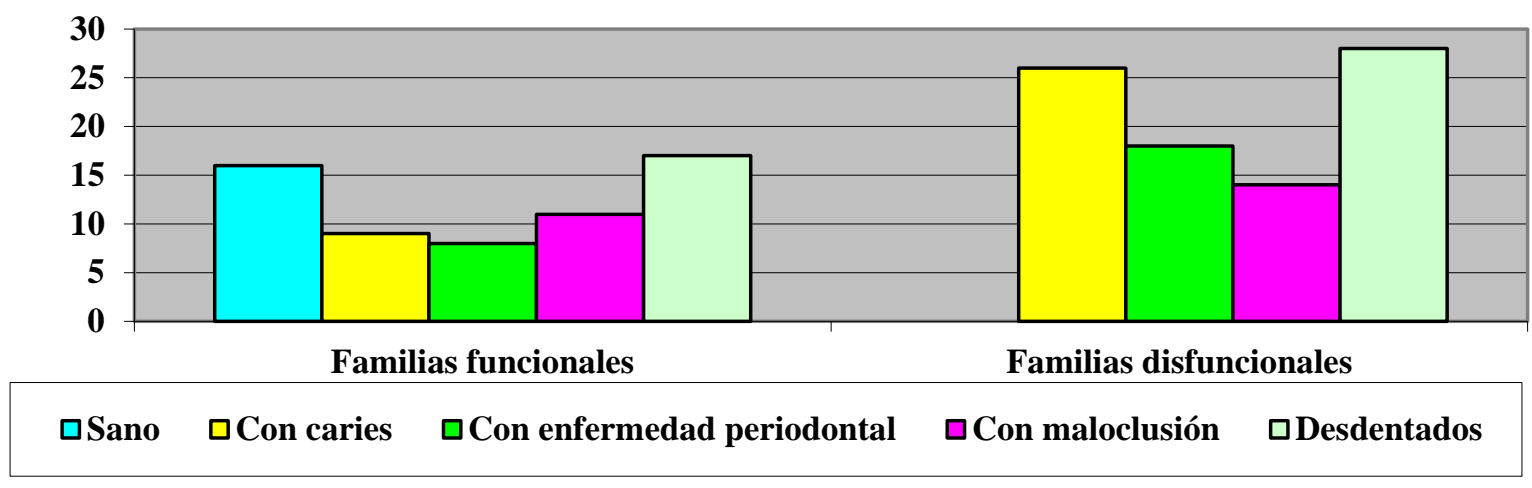

Los porcentajes de los problemas y/o enfermedades bucales se calcularon por los subtotales de columnas, y estos a su vez se calcularon en relación con el gran total $n=84$ (Chi cuadrado=28,7. $\mathrm{p}=0,0)$.

Del total de personas incluidas en el estudio, solo 7 (8.33 \%) no presentaban hábitos bucales perjudiciales, y se encontraban dentro de familias funcionales; el resto de los estudiados presentaron uno o más hábitos. 
FIGURA 2

DISTRIBUCIÓN DE PERSONAS, SEGÚN FUNCIONAMIENTO FAMILIAR Y LA PRESENCIA O AUSENCIA DE HÁBITOS DEFORMANTES

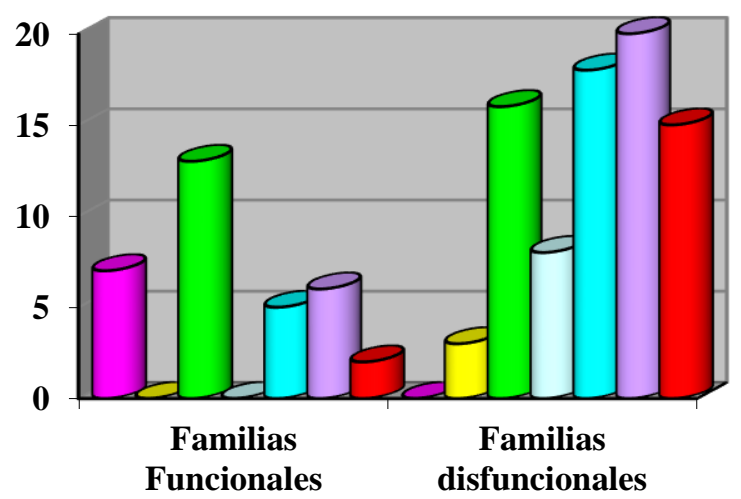

\begin{tabular}{lll}
\hline No presencia de hábitos & $\square$ Succión digital & $\square$ Respirador bucal \\
$\square$ Lengua protráctil & $\square$ Queilofagia & $\square$ Onicofagia \\
$\square$ Bruxismo & &
\end{tabular}

Los porcentajes de los hábitos bucales deformantes se calcularon por los subtotales de columnas, y estos a su vez se calcularon en relación con el gran total $n=84$ (Chi cuadrado=14,2. p=0,813).

En cuanto a la higiene bucal predominaron las personas con categoría de mala (59 para un 70.24 \%) siendo las más representadas (48 individuos para un $81.36 \%$ ). En cuanto a la buena higiene oral, aunque solo representó un 29.76 \%, la mayor representación estuvo en las personas de familias funcionales, con 22 integrantes con $88 \%$. 
FIGURA 3

DISTRIBUCIÓN DE SUJETOS, SEGÚN FUNCIONAMIENTO FAMILIAR Y LA HIGIENE BUCAL

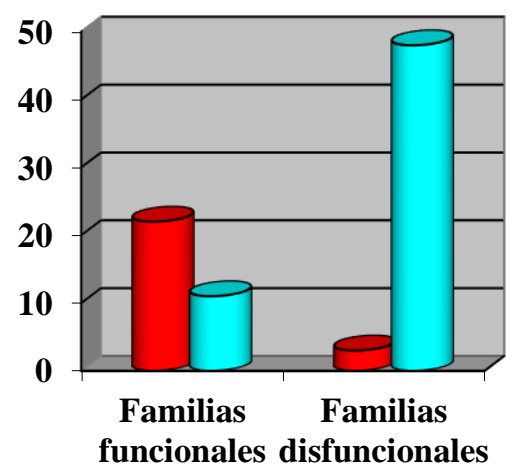

口Higiene bucal buena $\quad$ Higiene bucal mala

Los porcentajes de la higiene bucal se calcularon por los subtotales de columnas, y estos a su vez se calcularon en relación con el gran total $n=84$ (Chi cuadrado=24,5. $\mathrm{p}=0,000)$.

Respecto al cepillado dental el $51.19 \%$ de los miembros de los núcleos familiares estudiados se cepillaban 2 veces al día, la mayor representación estuvo en los integrantes de las familias disfuncionales con un $83.72 \%$. Solo el $10.71 \%$ se los cepillaban 4 veces al día, que es la frecuencia correcta del cepillado dental y todos pertenecían a familias funcionales. 
FIGURA 4

PORCENTAJE DE PERSONAS, SEGÚN FUNCIONAMIENTO FAMILIAR Y LA FRECUENCIA DEL CEPILLADO DENTAL.

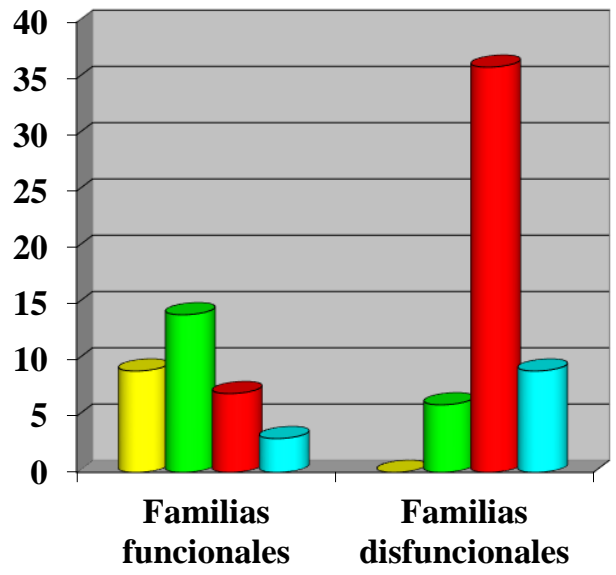

$\square$ Cepillado 4 veces/día $\square$ Cepillado 3 veces/día $\square$ Cepillado 2 veces/día $\square$ Cepillado 1 vez/día

Los porcentajes de las frecuencias del cepillado se calcularon por los subtotales de columnas, y estos a su vez se calcularon en relación con el gran total $n=84$ (Chi cuadrado=20,0. p=0,000).

La frecuencia del consumo de alimentos azucarados en las familias estudiadas se comportó de la forma siguiente: la mayoría de las personas consumían alimentos azucarados, sobre todo en las comidas $(83.33 \%)$, solo el $22.62 \%$ (19 integrantes) no consumían dieta rica en hidratos de carbono, siendo la mayoría miembros de núcleos familiares funcionales 15 para un 78,95\%. 
FIGURA 5

PORCENTAJE DE INDIVIDUOS SEGÚN FUNCIONAMIENTO FAMILIAR Y FRECUENCIA DE INGESTIÓN DE LOS ALIMENTOS AZUCARADOS

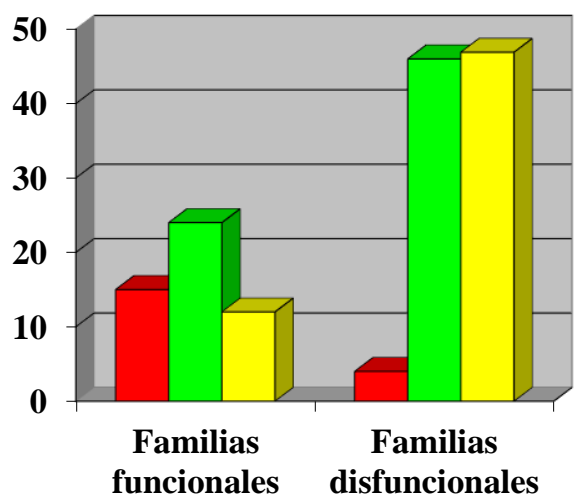

$\square$ No consumen alimentos azucarados

口Consumen alimentos azucarados en las comidas

Los porcentajes de las frecuencias de ingestión de alimentos azucarados se calcularon por los subtotales de columnas, y estos a su vez se calcularon en relación con el gran total $n=84$ (Chi cuadrado=13,1. $\mathrm{p}=0,000)$.

Más del $45 \%$ de las personas acudían a la consulta de Estomatología cuando tenían una urgencia (46.43\%), siendo los pertenecientes a las familias disfuncionales los más representados (82.05\%). Un pequeño grupo de 13 personas, para un $15.48 \%$ respondieron que iban al estomatólogo una vez al año, aunque no presentaran ninguna patología, y todos eran de familias funcionales. 
FIGURA 6

PORCENTAJE DE SUJETOS, SEGÚN FUNCIONAMIENTO FAMILIAR Y LA FRECUENCIA DE VISITAS A LA CONSULTA DE ESTOMATOLOGÍA

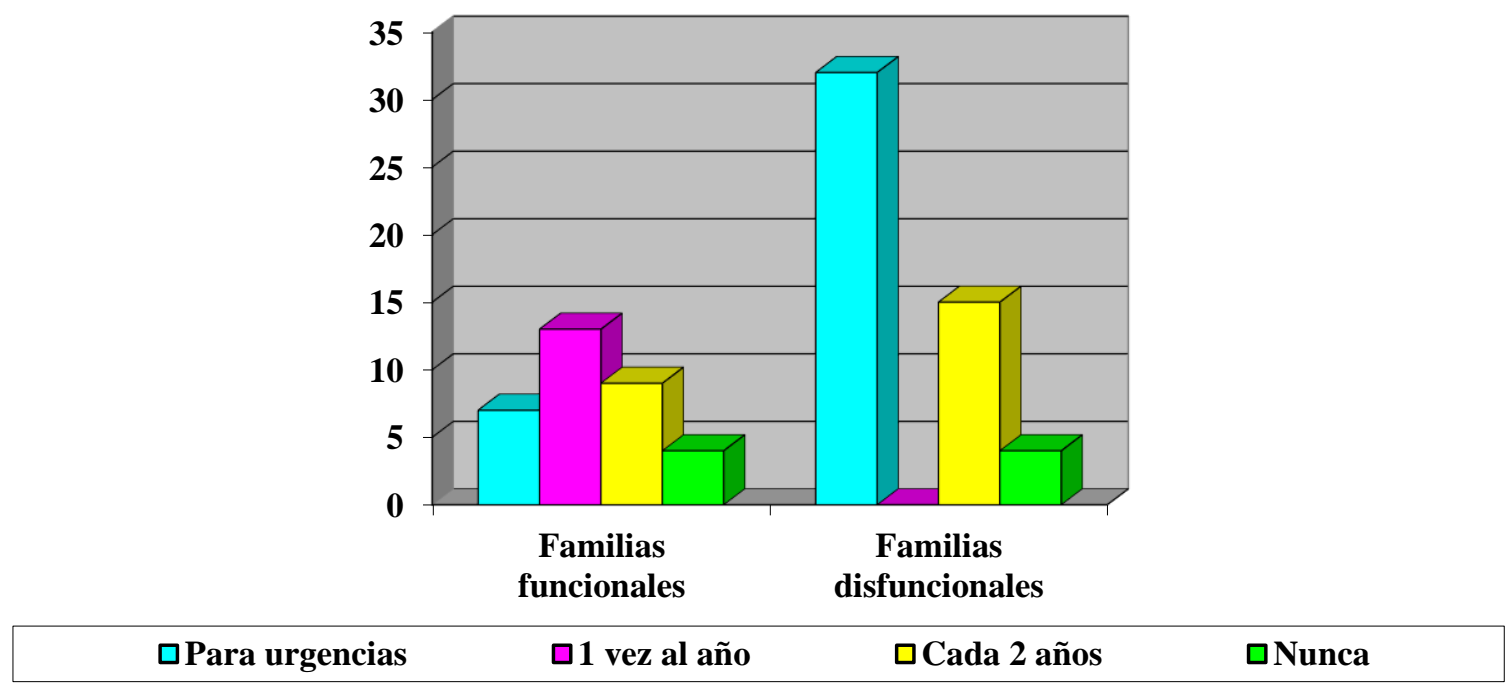

La frecuencia de las visitas a la consulta de estomatología se calculó por los subtotales de columnas. Estos, a su vez, se calcularon en relación con el gran total $n=84$ (Chi cuadrado=10,8. $\mathrm{p}=0,000)$.

\section{DISCUSIÓN}

El hecho de que la mayoría de las personas estudiadas pertenecieron a familias disfuncionales, es un dato a tener en cuenta a la hora de brindar atención estomatológica, pues se deben planificar actividades específicas de promoción y prevención de salud, encaminadas al logro de cambios en los estilos de vida de estos hogares; para mantener controlados toda una serie de factores de riesgo, 
como hábitos, costumbres y tradiciones familiares, que pudieran influir de forma directa o indirecta en la salud bucal de cada uno de los integrantes.

Se debe ver a la familia como fuente proveedora de salud, o de lo contrario, causante de enfermedad. La relación de la familia con el proceso salud-enfermedad de sus miembros, se pone de manifiesto en diversas investigaciones, tal es el caso de estudios realizados por investigadores cubanos en niños con alteraciones del retardo psíquico, donde se encontró un alto índice de disfuncionalidad en sus familias (35-37). Ya vinculado a la Estomatología, existen estudios, también en niños, que arrojaron una relación estadísticamente significativa, entre las enfermedades bucales, con las condiciones económicas de las familias, y con el nivel de educación de los padres (15-22). Otros trabajos (35-37) ponen en claro la necesidad de incrementar los conocimientos sobre temas de salud bucal en las familias.

La figura 2 muestra el edentulismo es un problema de salud bucal, que es el resultado de una larga historia de padecimientos de caries y/o enfermedad periodontal. A su vez este problema afecta las diferentes funciones del aparato estomatognático: estética, fonación, masticación, alteración de la articulación temporomandibular, entre otras; teniendo que recurrir, las personas a los servicios de prótesis en busca de solución a su problema. El desdentamiento, en otros trabajos realizados, estuvo relacionado con la caries dental, la enfermedad periodontal, las creencias erradas de la población respecto a este tema, y la falta de programas de promoción y prevención en las políticas de salud de sus países, cosa que no ocurre en Cuba $(32,33)$. No obstante a esto siguen altas las demandas en las consultas de prótesis en la población cubana. Estos resultados advierten una alta prevalencia de afecciones estomatológicas, lo que se traduce en una pobre educación para la salud y actitud preventiva en el núcleo familiar, sobre todo cuando existe disfuncionalidad. 
El resto de las enfermedades de la cavidad bucal en este estudio (32) tuvieron altos porcientos, lo que demuestra que las medidas preventivas, en el seno familiar no están siendo practicadas, tales como: cuidar que la embarazada tenga una correcta dieta, que incluya las vitaminas y minerales necesarios para la formación de los tejidos del futuro bebé (entre ellos los tejidos dentarios); una correcta higiene bucal, después de cada comida y antes de dormir, con la utilización de otros aditamentos que ayudan a la higiene (hilo dental, cepillos unipenacho); la ingestión de dietas ricas en alimentos protectores (frutas y vegetales) en la dieta diaria; la preocupación por parte de los padres de llevar a sus hijos, periódicamente a la consulta de estomatología $(32,33)$.

La familia debe velar porque dichas medidas se cumplan, sobre todo en los más jóvenes, para garantizar que lleguen a adultos sanos con todos sus dientes, evitándose las molestias que llegan a ocasionar las prótesis estomatológicas. Para reafirmar las razones expuestas anteriormente, existió una relación estadísticamente significativa entre el funcionamiento familiar con la presencia o ausencia de enfermedades y problemas de salud bucal. Diferentes autores coincidieron en que existe un desconocimiento en la familia respecto a lo concerniente a la Estomatología, por ejemplo: en la enfermedad periodontal y la caries, existió una relación altamente significativa ( $\mathrm{p}=0.000)$ entre una higiene bucal deficiente, aprendida en el seno familiar, y estas entidades patológicas (1526); estos autores además ven que existe un desconocimiento y despreocupación por parte de la población del riesgo de padecer estas enfermedades.

Las maloclusiones fueron encontradas frecuentemente en la población, sobre todo infantil, y dentro de los factores de riesgo figuran los hábitos deformantes: succión digital, empuje lingual, etc., y la 
pérdida prematura de dientes temporales y permanentes, por causa de caries dental, principalmente (15-19). Otros autores además de estos factores de riesgo, encontraron las malnutriciones en el embarazo y en la niñez (38-41).

En la figura 3 se muestra el hábito nocivo más frecuente fue el de respiración bucal con un total de 29 individuos para un $34.52 \%$, le siguieron cerca, en orden decreciente, la onicofagia (30.95 \%) y la queilofagia $(27.38 \%)$. Si se analiza según el funcionamiento familiar, en el orden de frecuencia de los hábitos bucales deformantes hubo variaciones en las familias disfuncionales, quedando de la forma siguiente: primero la Queilofagia (78.26\%), le siguen en orden descendente, la Onicofagia con un $76.92 \%$ y la respiración bucal con $55.17 \%$. Esta frecuencia de hábitos es diferente a la encontrada por otros investigadores $(40,41)$, lo que demuestra la individualidad de cada población, por lo que las acciones de salud deberán ser diferentes. Todos los miembros de las familias disfuncionales presentaron más de un hábito nocivo, observándose valores por encima del $50 \%$. Mientras que en las familias funcionales la mayoría de los integrantes solo poseían un hábito perjudicial.

El respirador bucal merece un comentario aparte, ya que es un paciente que no solo tiene problemas para respirar por la nariz, sino que esto va acompañado de signos y síntomas lo que lo hace un síndrome: Síndrome de Insuficiente Respirador Nasal (SIRN), esta forma incorrecta de respiración lleva no solo a maloclusiones dentarias sino también a afectaciones ortopédicas, auditivas, retardo en el aprendizaje, entre otras $(40,42)$. Este hábito debe ser detectado por la familia para acudir al profesional en busca de las causas para erradicarlo, y evitar así que siga 
produciendo anomalías en todo el cuerpo, sobre todo cuando el niño está en la etapa del desarrollo $(40,42,43)$.

En el caso de la onicofagia y la queilofagia, son hábitos nocivos que se han visto asociados a estrés generados por conflictos en los diferentes escenarios donde se desenvuelve el individuo: hogar, escuela, centro de trabajo; por lo que es de vital importancia detectar las causas que originaron, o que pudieran originar el hábito para de esta forma actuar en ellas y lograr su eliminación; precisamente esta constituye una de las medidas preventivas en Estomatología. El Estomatólogo General Integral debe trabajar con la embarazada, las madres con niño menores de un año y con niños de edad escolar; además con las educadoras y maestras, de manera de capacitarlos, para evitar que se instale el hábito y en el caso de que ya exista, poder tratarlo a tiempo.

Este análisis coincide con otras investigaciones llevadas a cabo (34, 39-44), en las que se detectaron una alta incidencia de hábitos bucales deformantes en niños de círculo infantil, provocada por una inadecuada cultura sanitaria de los padres, acciones de salud ineficaces y poca sensibilización del personal del círculo infantil con los problemas de salud bucal; además coinciden en que es importante el control y eliminación de estos hábitos en edades tempranas, evitando de esta forma maloclusiones. A pesar que en esta investigación, no existió una relación estadísticamente significativa ( $\mathrm{p}=0.813$ ), entre le funcionamiento familiar y la presencia o no de hábitos bucales deformantes en sus miembros, se concuerda con los estudios realizados por diferentes autores, entre ellos Bayardo (34), en los que especifican que la familia puede intervenir favoreciendo la eliminación de estos hábitos, o de lo contrario, perpetuándolos (11, 13, 42, 43). 
En la figura 4 se describe cómo la higiene bucal es un hábito que comienza en el hogar, es trasmitido de una generación a otra como parte de la higiene personal. Se observó a través de los resultados obtenidos como la familia dentro de sus múltiples funciones, entre ellas la educación, va educando a sus integrantes e inculcando en ellos, los estilos de vida que han trascendido a lo largo de su generación, aunque estos no sean los más favorecedores para la salud.

La población necesita conocer más sobre aspectos relacionados con la higiene bucal, en primer lugar, la importancia que tiene el cepillado dental como medida preventiva y eficaz, no solo para evitar enfermedades sino para que el individuo disfrute del placer de una boca limpia, de una higiene bucal correcta; lo que producirá un doble efecto: bienestar y prevención de las enfermedades más frecuentes de la Estomatología, evitando la pérdida dentaria que tanto afecta las diversas funciones del aparato estomatognático, entre ellas la estética.

Aspectos tales como la frecuencia correcta para la realización de este, es desconocido todavía por muchas personas, no saben que deben cepillarse antes de dormir ni la importancia de esto. En el caso de personas portadoras de prótesis parciales removibles, existe la creencia de que los retenedores "ganchitos" le producen caries dental, cuando en realidad lo que provoca la aparición de dicha patología es el incorrecto cepillado de las prótesis y de sus retenedores, así como los dientes remanentes. Es aquí donde entra a actuar la ardua labor educativa del Estomatólogo General Integral, brindando toda la información que necesita la población respecto a su salud bucal; si bien es cierto que es el individuo el máximo responsable de su salud, el especialista debe brindarle las herramientas necesarias para mantenerlo sano (el conocimiento). 
Coincidentemente otros autores, sobre todo de América Latina, han demostrado que el cepillado dental no es practicado correctamente por la población, existiendo un desconocimiento en cuanto a forma y frecuencia del mismo y la no utilización del hilo dental como aditamento para ayudar más a la higiene bucal. Además estos estudios sacan a la palestra pública, la no existencia, en sus países, de políticas de salud encaminadas a la promoción, prevención, así como a la accesibilidad y la gratuidad de los servicios de salud, cosa que caracteriza a las ciencias médicas de Cuba (15, 20, 33). Las variables en este caso tuvieron una asociación altamente significativa ( $\mathrm{p}=0.000)$, lo que corrobora una vez más, el papel de la familia como formadora de hábitos beneficiosos para la salud, con la ayuda del profesional (estomatólogo).

En la figura 5, la calidad del cepillado dental (frecuencia, forma, tiempo que se le dedica) es directamente proporcional a la higiene bucal, es decir que mientras más veces se realice, de forma correcta, y más tiempo se le dedique al cepillado mayor probabilidad de controlar la placa dentobacteriana y por lo tanto una correcta higiene bucal. Es en el seno familiar, donde los adultos comienzan a enseñar el cepillado a los pequeños, y se lo deben practicar hasta que el niño tenga dominio de sus movimientos, y se lo realice solo. Si los adultos no se cepillan correctamente, serán transmisores de un cepillado incorrecto. Las personas que refirieron cepillarse solo 2 veces al día, eliminaban la de después de almuerzo y la de antes de dormir; por lo tanto era mayor el tiempo de permanencia de la placa dentobacteriana, con restos de alimentos, sobre la superficie de los dientes. Esto se corresponde con otros datos obtenidos, sobre todo en las familias disfuncionales, donde existió un predominio de higiene bucal mala y enfermedades bucales en sus miembros. 
Es responsabilidad del estomatólogo enseñar a sus pacientes la frecuencia correcta del cepillado, sugiriendo algunas medidas para cuando no se pueda realizar en los centros de trabajo e instituciones escolares, tales como: enjuagarse la boca después de almuerzo, tratar de no consumir alimentos azucarados en este período de tiempo y cuando lleguen a sus hogares cepillarse inmediatamente.

Otros investigadores $(23,33,35,44)$ coinciden en que el correcto cepillado dental es un factor protector de enfermedades como la caries dental y la enfermedad periodontal, y de esta última no solo la evita sino que disminuye el riesgo de fracasos en su tratamiento. Ponen además de manifiesto que la familia cumple un rol fundamental en la instauración de correctos hábitos de higiene bucal en todos y cada uno de sus miembros y que debe velar por que así se cumplan, sobre todo en los más jóvenes cuya personalidad se está formando. Enfatizando la veracidad de estos resultados, al realizar la prueba estadística dio la existencia de una relación significativa entre la frecuencia del cepillado dental de los sujetos con la funcionalidad de sus familias.

En la figura 6, esta forma de consumir estos alimentos constituye un factor de riesgo de enfermedades como la diabetes mellitus, obesidad y la caries dental; esta última fue una de las afecciones más frecuentes encontrada en las personas de familias disfuncionales, coincidiendo con el hecho de que estos mismos sujetos son los mayores consumidores de hidratos de carbono. En el lactante todavía no se ha desarrollado el sentido del gusto son los adultos que rodean al pequeño y que se encargan de la elaboración de sus alimentos, los que comienzan a incorporar los diferentes condimentos, educando así el paladar de los pequeños. 
No se trata de eliminar completamente el azúcar de la dieta, sino que esta sea administrada con medida y en horarios donde seguidamente se proceda a realizar el cepillado dental. Es una tarea bien difícil cambiar hábitos dietéticos perjudiciales, pero no imposible, es necesario que el Estomatólogo incentive en la población la necesidad del cambio, para esto debe emplear buenas razones de manera que los individuos sean capaces de comprender y lo realicen. Para llevar a cabo esta misión es necesario una buena comunicación entre el Estomatólogo y la comunidad, a través de diferentes técnicas afectivas participativas, utilizando personas de la comunidad, de esta manera se pone de manifiesto uno de los principios de la promoción de salud, que es la participación comunitaria en la resolución de sus propios problemas de salud.

El consumo de azúcares en exceso en la dieta diaria también fue encontrado con gran frecuencia por otros investigadores, los cuales plantean la necesidad de erradicar o disminuir la ingestión de estos, ya que existe una tendencia a elevarse los índices de caries en la medida que aumenta su consumo; recomiendan que deben ser sustituidos por otros no cariogénicos como frutas y vegetales $(22,28,35-38)$. En esta investigación la relación entre el funcionamiento familiar y la frecuencia de ingestión de alimentos azucarados resultó estadísticamente significativa $(\mathrm{p}=0.000)$. Entre las razones que más refirieron los estudiados, del por qué no acudían a la consulta de Estomatología, estuvo el temor por este servicio.

Existe en la población un temor o miedo generalizado hacia la atención estomatológica, trayendo como consecuencia la no asistencia a estos servicios. Este miedo se va trasmitiendo dentro del núcleo familiar a otras generaciones más jóvenes lo que trae como resultado actitudes negativas 
hacia la Estomatología, provocando que las personas solo acudan cuando ya no puedan dilatar más la espera, que es el caso de las urgencias: dolor insoportable, fracturas, sangramientos, inflamaciones o para resolver rápidamente (es el caso de las exodoncias) y no vuelven a consulta hasta que se le presenta otra urgencia, lo cual los lleva necesariamente a un deterioro de su salud bucal, con la consecuente mortalidad dentaria y el aumento de las necesidades de prótesis. Esto está muy relacionado con el problema que más se detectó en los individuos, que fue el desdentamiento.

Existieron personas, aunque un pequeño porciento, que respondieron que nunca iban al estomatólogo porque eran portadores de prótesis; esto es incorrecto lo hacen por desconocimiento, pues aunque la persona no tenga dientes naturales debe acudir al estomatólogo para revisión de las estructuras que están en contacto con la prótesis, en busca de lesiones que pudieran ser la antesala del cáncer bucal, siendo esta otras de las medidas preventivas en Estomatología. Es una obligación y deber, por parte de los profesionales de la salud, de educar a la población sobre temas de Estomatología que todavía desconocen, para de esta manera erradicar las actitudes negativas y prejuiciosas hacia nuestra especialidad.

En la bibliografía consultada no se encontraron trabajos que reflejaran la relación del funcionamiento familiar con la frecuencia de visitas a la consulta de Estomatología, sin embargo de forma somera en tres artículos se pone de manifiesto que existe un predominio de las personas que solo utilizan los servicios estomatológicos para resolver una urgencia (22, 28, 35-38). A través de estos resultados se puede apreciar el lugar que ocupa la atención estomatológica en la vida de las familias con diferente funcionamiento familiar. 
El valor del estadígrafo Chi cuadrado resultó altamente significativo, lo que sugiere que existió asociación significativa entre estas dos variables $(\mathrm{p}=0.000)$.

\section{CONCLUSIONES}

El edentulismo fue el problema de salud bucal de mayor frecuencia, siendo los individuos de familias disfuncionales los más representativos.

Los hábitos bucales deformantes mayormente detectados fueron: respiración bucal, onicofagia y queilofagia; no existió relación estadísticamente significativa entre estos hábitos con el funcionamiento familiar.

Las familias disfuncionales tuvieron la mayor cantidad de personas con higiene bucal mala.

Más de la mitad de las personas se cepillaban dos veces al día, los integrantes de núcleos familiares disfuncionales fueron mayormente representados.

La mayoría de las personas consumían alimentos azucarados en las comidas, sobre todo aquellas de familias disfuncionales.

Más de un tercio de los miembros de las familias estudiadas, acudían a la consulta estomatológica cuando presentaban una urgencia, siendo la mayoría de núcleos familiares disfuncionales. 
Existió relación entre el funcionamiento familiar y el estado de salud bucal de sus integrantes.

\section{RECOMENDACIONES}

Realizar un mayor trabajo comunitario con las familias disfuncionales, vinculando el sector salud con los diferentes factores de la comunidad, para elaborar estrategias conjuntas, que permitan la promoción de salud y la prevención de enfermedades en estas familias.

\section{REFERENCIAS}

1. Louro I. La familia en la determinación de la salud. Rev Cubana Salud Pública.2003 ene-mar; $1(29)$.

2. Editorial Félix Varela. Psicología de la Familia: Una aproximación a su estudio. La Habana; 2002.

3. Editorial Científico-Técnica. Para que la Familia funcione bien. La Habana; 2005.

4. Editorial de Ciencias sociales. Mi familia es así. La Habana; 1990.

5. Editorial Pueblo y Educación. Hacia el perfeccionamiento de la escuela primaria. La Habana; 2002.

6. Rodríguez A, Valiente C, Tan N. Las familias saludables y los factores determinantes del estado de salud. Rev Cubana Estomatol. 2001 sep-dic; 3(38): 10-2. 
7. Pérez C, Fernández OC. Evaluación de un programa de intervención familiar educativa. Rev Cubana Med Gen Integr. 2005 ene-abr; 1-2(21): 4-7.

8. Santos AP, Soviero VM. Caries prevalence and risk factors among children aged 0 to 36 months. Pesqui Odontol Bras. 2002 Jul-Sep;16(3): 203-8.

9. Polido K, Petinuci A, Castanha JF, Magalhães JR. Prevenção de cárie dentária e doença periodontal em Ortodontia: uma necessidade imprescindible. Rev Dent Press Ortodon-Ortop Facial. 2006 mar-abr; 2(11): 11-7.

10. Boing AF, Peres MA, Douglas F, Zange SE, Ferreira JL. Prevalência de cárie e fatores associados em crianças da comunidade do Vietnã, Recife. Rev Bras Saude Mater Infant. 2005 abr-jun; 2(5):5-10.

11. Duque de Estrada J, Rodríguez A, Coutin G, Riveron F. Factores de riesgos asociados con la enfermedad caries dental en niños. Rev Cubana Estomatol. 2003 mayo-ago; 2(40):6-10.

12. Duque de Estrada J, Rodríguez A, Coutin G, Riveron F. Factores de riesgos asociados con la enfermedad periodontal en niños. Rev Cubana Estomatol. 2003; 40(1):11-6.

13. Felipe S. Relación entre el nivel de conocimientos sobre higiene bucal y la salud gingival en la adolescencia [tesis]. Ciudad Habana: Facultad de Estomatología; 2002.

14. Love D et al. An oral hygiene measurement system for possible research an use. J Public, Match. Dent Periodontitis. 2006; 5(32)120-4.

15. Ferreira JL, Capel P. Perda dentária precoce em adultos de 35 a 44 anos de idade. Rev bras epidemiol. 2003 abr; 1(6): 34-7.

16. Romero Y. Hábitos que influyen el proceso salud-enfermedad bucal. Estudio etnográfico en grupo de madres de la comunidad de San Isidro, estado de Mérida, 2003. Acta odontol venez. 2006 ene; 1(44) primera parte. 
17. Fernández CM, Acosta A. Hábitos deformantes en escolares de primaria. Rev cuba ortod. 1997; 12(2): 79-83.

18. Rojas R, Báez J, Rojas R. Prevalencia de malos hábitos orales y respiración bucal en niños de 5 a 17 años del área de Santiago Centro. Rev Fac odontol. 2001; 19 (1): 9-19.

19. Louro I. Matriz de salud del grupo familiar: un recurso para el diagnóstico de la situación de salud de la familia. Rev Cubana Med Gen Integr. 2004 Mayo-Jun; 3(20):23-6.

20. González I. Reflexiones acerca de la salud familiar. Rev Cubana Med Gen Integr. 2000 septoct; 5(16):12-6.

21. Herrera P. Rol de género y funcionamiento familiar. Rev Cubana Med Gen Integr. 2000 novdic; 6(16):5-10.

22. Louro I. Modelo de salud del grupo familiar. Rev Cubana Salud Pública, Ciudad de La Habana. 2005 sep.-dic; 4(31):8-12.

23. Louro I. Algunas consideraciones acerca de la familia en el proceso Salud Enfermedad. Experiencia en Atención Primaria de Salud, 1993. Rev Cubana Salud Pública. 2000 ene-jun; 1(26):5-8.

24. Carvalho B, Cople L. Cárie tipo mamadeira e a importância da promoção de saúde bucal em crianças de 0 a 4 anos. Rev Odontol Univ São Paulo. 1999 jul-set; 3(13):21-7.

25. Saito SK, Subvertí HM, dos Santos MN. Efeito da prática de alimentação infantil e de fatores associados sobre a ocorrência da cárie dental em pré-escolares de 18 a 48 meses. Rev Odontol Univ São Paulo. 1999 ene-mar; 1(13): 112-6.

26. Tascón JE, Cabrera G. Creencias sobre caries e higiene oral en adolescentes del Valle del Cauca. Rev Colomb Med. 2005; 36: 73-78. 
27. Heberto F, Suárez C, de León I, Rivera DM. Eliminación de factores de riesgo de maloclusión dentarias en niños de primaria. Rev Cubana Estomatol. 2002; 2 (41):5-9.

28. Podadera ZR, Ruiz D. Prevalencia de hábitos deformantes y anomalías dentomaxilofaciales en niños de 3 a 6 años de edad, 2002-2003. Rev Cubana Estomatol. 2004 may-ago; 2(41):12-7.

29. Duque de Estrada Y, Rodríguez A, Coutin G, González N. Factores de riesgo asociado con la Maloclusión. Rev Cubana Estomatol. 2004; 23 (1):4-7.

30. Alfonso NM, Martínez T, Pría MC, Roche A, García A. Salud bucal de la población. Policlínicos "Plaza de la Revolución" y "Héroes del Moncada", 1999-2001. Rev Cubana Estomatol. 2004; (1).

31. Fernández CM, Acosta A. Estado actual de la atención a escolares de primaria. Rev cuba ortod. 1997; 12 (2): 91-5.

32. Bravo D, de León I, Llorach J, Chaviano M. Intervención educativa en hábitos bucales deformantes en el Círculo Infantil "Amores de la Patria". Rev Med Matanzas. 2004; 21(5):116.

33. Ortega G, Rodríguez R, Negrín V. Hábitos bucales incorrectos de succión al año de edad. Rev cuban ortod. 1993; 81(1):47-9.

34. Bayardo F. Los malos hábitos orales en niños. Etiología. Rev A D M. 1995; 52(2): 79-84.

35. Romero Y. Hábitos que influyen el proceso salud-enfermedad bucal. Estudio etnográfico en grupo de madres de la comunidad de San Isidro, estado de Mérida, 2003. Acta Odontol Venez. 2006 ene; 1(44) segunda parte.

36. Pires AP, Canano M, Barbosa ME, Mendes V. Frequiência de higiene bucal e presença de biofilme visível na dentição decídua. Braz oral. 2007 ene-mar; 1(21):24-9. 
37. Fejerskov O, Kidd E. Dental caries - The disease and its clinical management. Copenhagen: Blackwell Munksgaard. 2003:171-7.

38. Sayegh A, Dini EL, Holt RD, Bedi R. Oral health, sociodemographic factors, dietary and oral hygiene practices in Jordanian children. J Dent. 2005; 33(5):379-88.

39. Prado JS, Aquino DR, Cortelli JR, Cortelli SC. Condição dentária e hábitos de higiene bucal em crianças com idade escolar [on line]. 2001.

40. Peres MA, Peres KG, Antunes JL, Junqueira SR, Frazão P, Narvai PC. The association between socioeconomic development at the town level and the distribution of dental caries in Brazilian children. Rev Panam Salud Pública. 2003; 14:149-57.

41. Cangussu CT, Castellanos RA, Pinheiro MF, Albuquerque SR, Pinho C. Cárie dentária em escolares de 12 e 15 anos de escolas públicas e privadas de Salvador do Bahia, Brasil, em 2001. Pesqui Odontol Bras. 2002; 16:379-84.

42. Andrade T, Sabioni M, Almeida R, Gonçalves IC. Cárie dental e consumo de açúcar em crianças assistidas por creche pública. Rev Odontol Univ São Paulo. 1999 ene-mar; 1(13):208.

43. Blanco J. Consumir azúcar con moderación. Rev cuban aliment nutr. 2002; 16(2):142-5.

44. Ortiz A. Condiciones de vida y de salud bucal del escolarizado y su familia. Municipio Caroni, Estado Bolívar, 1992. Acta odontol venez. 2000 ene; 1(38):10-7. 


\section{APÉNDICE 1}

TEST DE FUNCIONAMIENTO FAMILIAR

A continuación le presentamos una serie de situaciones que pueden ocurrir o no en su familia. Necesitamos que Usted clasifique y marque con una $\mathbf{X}$ su respuesta según la frecuencia en que ocurre la situación.

\begin{tabular}{|c|c|c|c|c|c|c|}
\hline Ítem & Situaciones familiares & $\begin{array}{c}1 \\
\text { Casi } \\
\text { nunca }\end{array}$ & $\begin{array}{c}2 \\
\text { Pocas } \\
\text { veces }\end{array}$ & $\begin{array}{c}3 \\
\text { A veces }\end{array}$ & $\begin{array}{c}4 \\
\text { Muchas } \\
\text { veces }\end{array}$ & $\begin{array}{c}5 \\
\text { Casi } \\
\text { siempre }\end{array}$ \\
\hline 1 & $\begin{array}{l}\text { Se toman decisiones para cosas importantes de la } \\
\text { familia considerando la opinión de todos. }\end{array}$ & & & & & \\
\hline 2 & En mi casa predomina la armonía. & & & & & \\
\hline 3 & $\begin{array}{l}\text { En mi familia cada uno cumple sus } \\
\text { responsabilidades. }\end{array}$ & & & & & \\
\hline 4 & $\begin{array}{l}\text { Las manifestaciones de cariño forman parte de } \\
\text { nuestra vida cotidiana. }\end{array}$ & & & & & \\
\hline 5 & $\begin{array}{l}\text { Nos expresamos sin insinuaciones, de forma } \\
\text { clara y directa. }\end{array}$ & & & & & \\
\hline 6 & $\begin{array}{l}\text { Podemos aceptar los defectos de los demás y } \\
\text { sobrellevarlos. }\end{array}$ & & & & & \\
\hline 7 & $\begin{array}{l}\text { Tomamos en consideración las experiencias de } \\
\text { otras familias ante situaciones difíciles. }\end{array}$ & & & & & \\
\hline 8 & $\begin{array}{l}\text { Cuando alguno de la familia tiene un problema, } \\
\text { los demás lo ayudan. }\end{array}$ & & & & & \\
\hline 9 & $\begin{array}{l}\text { Se distribuyen las tareas de forma que nadie esté } \\
\text { sobrecargado. }\end{array}$ & & & & & \\
\hline 10 & $\begin{array}{l}\text { Las costumbres familiares pueden modificarse } \\
\text { ante determinadas situaciones. }\end{array}$ & & & & & \\
\hline 11 & Podemos conversar diversos temas sin temor. & & & & & \\
\hline 12 & $\begin{array}{l}\text { Ante una situación familiar difícil somos capaces } \\
\text { de buscar ayuda en otras personas. }\end{array}$ & & & & & \\
\hline 13 & $\begin{array}{l}\text { Los intereses y necesidades de cada cual es } \\
\text { respetado por el núcleo familiar. }\end{array}$ & & & & & \\
\hline 14 & Nos demostramos el cariño que nos tenemos. & & & & & \\
\hline
\end{tabular}

Marque con una $\mathrm{X}$ el tiempo que hace que su familia es así:

Menos de 1 año.

Entre 1 y 2 años.

Más de 2 años.

Clasificación de la Familia según funcionamiento familiar (marcar con una X):

Familia funcional ---

Familia disfuncional ---- 
APÉNDICE 2

ÍNDICE DE ANÁLISIS DE HIGIENE DE LOVE (IAHB)

\begin{tabular}{|l|l|l|l|l|l|l|l|l|l|l|l|l|l|}
\hline & & & & & & & & & & & & & \\
\hline $\mathbf{7}$ & $\mathbf{6}$ & $\mathbf{5}$ & $\mathbf{4}$ & $\mathbf{3}$ & $\mathbf{2}$ & $\mathbf{1}$ & $\mathbf{1}$ & $\mathbf{2}$ & $\mathbf{3}$ & $\mathbf{4}$ & $\mathbf{5}$ & $\mathbf{6}$ & $\mathbf{7}$ \\
\hline & & & & & & & & & & & & & \\
\hline
\end{tabular}

$\mathrm{IAHB}=\underline{\text { Superficies coloreadas }} \times 100 \%$

Índice de Análisis de Higiene de Love: Es un índice adecuado para la investigación y uso clínico. Refleja, en forma precisa, la habilidad del paciente para controlar su placa dentobacteriana, y eliminar restos de alimentos de toda la superficie dentaria. Sirve para poder evaluar la eficiencia de los distintos métodos y medios que utilizan los pacientes para el control de su placa dentobacteriana. Como unidad, este índice, toma la superficie total de cada cara del diente. Se utiliza una sustancia reveladora de placa dentobacteriana, lo que reduce grandemente la subjetividad del observador; se califica con 0 -si la superficie está limpia y con 1-si la superficie está sucia; para obtener el valor de este índice es por el número de superficies dentarias manchadas entre el número de superficies examinadas multiplicado por 100, se da en porciento. La interpretación es: se considera que el paciente tiene una higiene bucal buena cuando el valor del índice está entre el $14 \%$ y el $20 \%$.

\section{APÉNDICE 3}

\section{FORMULARIO SOBRE SALUD BUCAL FAMILIAR}

\section{1) Datos Generales de la familia}

N. ${ }^{\circ}$ de la familia:

Dirección de la vivienda:

Nombre (s) y apellidos de cada uno de los integrantes que viven bajo el mismo techo, y parentesco en relación al jefe de núcleo.

\begin{tabular}{|l|l|l|}
\hline $\begin{array}{l}\text { No. del } \\
\text { integrante }\end{array}$ & Nombre (s) y Apellidos & Parentesco \\
\hline 1 & & \\
\hline 2 & & \\
\hline 3 & & \\
\hline
\end{tabular}

\section{Examen Bucal}

a) Presencia o ausencia de problemas y/o enfermedades bucales en cada uno de los miembros del núcleo familiar (marcar con una $\mathrm{X}$ )

\begin{tabular}{|l|l|l|l|l|l|}
\hline $\begin{array}{l}\text { No. del } \\
\text { integrante }\end{array}$ & Sano & Caries & $\begin{array}{l}\text { Enfermedad } \\
\text { Periodontal }\end{array}$ & Maloclusión & Desdentados \\
\hline 1 & & & & & \\
\hline 2 & & & & & \\
\hline 3 & & & & & \\
\hline
\end{tabular}

b) Presencia o ausencia de hábitos bucales deformantes (marcar con una X)

\begin{tabular}{|l|l|l|l|l|l|l|l|}
\hline $\begin{array}{l}\text { No. del } \\
\text { integrante }\end{array}$ & $\begin{array}{l}\text { No } \\
\text { presen } \\
\text { cia }\end{array}$ & $\begin{array}{l}\text { Succión } \\
\text { digital }\end{array}$ & $\begin{array}{l}\text { Respirador } \\
\text { bucal }\end{array}$ & $\begin{array}{l}\text { Lengua } \\
\text { protráctil }\end{array}$ & Queilofagia & Onicofagia & $\begin{array}{l}\text { Bruxis } \\
\text { mo }\end{array}$ \\
\hline 1 & & & & & & & \\
\hline 2 & & & & & & & \\
\hline 3 & & & & & & & \\
\hline
\end{tabular}


c) Higiene bucal según índice de Love (marcar con una X)

\begin{tabular}{|l|l|l|}
\hline $\begin{array}{l}\text { No. } \\
\text { integrante del }\end{array}$ & HB buena & HB mala \\
\hline 1 & & \\
\hline 2 & & \\
\hline 3 & & \\
\hline
\end{tabular}

d) Frecuencia del cepillado dental de cada miembro del núcleo familiar (marcar con una X)

\begin{tabular}{|l|l|l|l|l|}
\hline $\begin{array}{l}\text { No. } \\
\text { integrante del }\end{array}$ & 4 veces/día & 3 veces/día & 2 veces/día & 1 vez/día \\
\hline 1 & & & & \\
\hline 2 & & & & \\
\hline 3 & & & & \\
\hline
\end{tabular}

e) Frecuencia de la ingestión de alimentos azucarados (marcar con una X)

\begin{tabular}{|l|l|l|l|}
\hline $\begin{array}{l}\text { No. del } \\
\text { integrante }\end{array}$ & No consume & En las comidas & Entre comidas \\
\hline 1 & & & \\
\hline 2 & & & \\
\hline 3 & & & \\
\hline
\end{tabular}

f) Frecuencia de visitas a la consulta Estomatológicas de los integrantes del núcleo familiar (marcar con una X)

\begin{tabular}{|l|l|l|l|l|}
\hline $\begin{array}{l}\text { No. del } \\
\text { integrante }\end{array}$ & Para urgencias & 1 vez al año & Cada 2 años & Nunca \\
\hline 1 & & & & \\
\hline 2 & & & & \\
\hline 3 & & & & \\
\hline
\end{tabular}

